\title{
Frequency of myasthenia gravis in multiple sclerosis: Report of five cases from Isfahan, Iran
}

\author{
Keivan Basiri, Masoud Etemadifar, Amir Hadi Maghzi, Neda Zarghami \\ Department of Neurology, Isfahan University of Medical Sciences, Alzahra Hospital, Sofe Ave, Isfahan, Iran
}

\author{
Address for correspondence: \\ Dr. Keivan Basiri, \\ Department of Neurology, Alzahra \\ Hospital, Isfahan University of \\ Medical Sciences, \\ Sofe Ave, Isfahan, Iran. \\ E-mail: basiri@med.mui.ac.ir
}

PMID: 19934567

DOI: $10.4103 / 0028-3886.57817$

\begin{abstract}
This study was designed to determine the frequency and clinical characteristics of myasthenia gravis (MG) in a large cohort of Persian patients with multiple sclerosis (MS) living in the province of Isfahan. We reviewed the case records of patients with definite MS (McDonald's criteria) registered in the Isfahan MS Society (IMSS) for associated MG. Of the 1,718 patients with MS in the registry, six patients were found to have both MS and MG. The prevalence after excluding one patient with insufficient data, was 29 I per 100,000 (0.29\%), a higher prevalence than the earlier reports. These results may support the hypothesis that MS and MG share some common immunopathogenic mechanisms.
\end{abstract}

Key words: Myasthenia gravis, multiple sclerosis, repetitive nerve stimulation

\section{Introduction}

Myasthenia gravis (MG) and multiple sclerosis (MS) are both autoimmune disorder, but with differing target organs, MS involves myelin sheath while MG involves the neuro-muscular junction. There are some shared commonalities between MS and MG, like the age and sex distribution, and associations with HLA types (DR3, B8, A1, and A2). ${ }^{[1]}$ This study was designed to determine the frequency and clinical characteristics of MG in a large cohort of Persian patients with MS living in the province of Isfahan.

\section{Materials and Methods}

We reviewed the case records of 1,718 (1,330 females and 388 males) patients with definite MS based on the McDonaled criteria, registered in the Isfahan MS society (IMSS) from April 2003 to July 2006. ${ }^{[2]}$ Patients with fluctuating signs and symptoms suggestive of MG were included in the study. All identified patients were interviewed and examined by a neurologist to obtain the clinical and demographic information. Diagnosis of MG was based on clinical features, repetitive nerve stimulation (RNS), edrophonium (Tensilon) test, and detection of acetylcholine receptor antibody (Ach-R antibody) in the serum. RNS was performed with a Medelec Synergy system and a decrement more than $10 \%$ (reduction of CMAP amplitude more than $10 \%$ with $3 \mathrm{~Hz}$ stimulation rate) was considered significant. Edrophonium test was performed by administration of ten milligram (mg) of edrophonium (in three bolus: 2,3 , and $5 \mathrm{mg}$ respectively) through a peripheral vein and consequent objective improvement of signs was considered as a positive response. All tests were performed by a qualified neurologist.

\section{Results}

Of the 1,718 patients with definite MS registered in IMSS, 6 patients were identified to have both MS and MG. One of the patients had migrated to another area and was excluded for calculating the prevalence rates as he was unavailable for further interview. This gave a prevalence of 291/100,000 $(0.29 \%)$ of MG among patients with definite MS. The diagnosis of MG was supported by clinical features, positive edrophonium test and presence of significant decrement response in all the six patients. None of the patients had positive Ach-R antibody serology. 
All the five patients were females and less than 55 years of age at the time of diagnosis. The mean-age was 37.4 (range, 25-55). The mean age at the onset of first MS symptom was 32.5 (range: 19-51) and the mean age of initial MG symptom was 33.4 (range: 16-52) [Table 1].

First symptom of MS was optic neuritis in two patients, limb paresthesia in another two, and facial palsy in the remaining one patient. In three patients MS occurred before MG (1-9 years, mean 3 years) and in two, MS occurred after MG (6 months-7 years; mean 4 years). The initial MG symptoms were ocular (ptosis and dipplopia) in three patients, ocular and bulbar (ptosis and dysartheria) in one, and bulbar (fluctuating dysphonia) in the other. There were severe fluctuations in the weakness in all the patients. The course of MS in all the five patients was relapsing-remitting. The severity of both MG and MS was mild in all patients (EDSS scores varied from 1 to 2.5) [Tables 1 and 2].

\section{Discussion}

The prevalence of MG among the patients with definite MS in our study was significantly higher, 291/100000, when compared to the reported prevalence of MG in the general population, 5-15/100000. ${ }^{[3,4]}$ In earlier reports there were 35 patients with a diagnosis of both central primary demylinating disease and MG.$^{[5-11]}$ The central primary demyelinating disease among these 35 patients was MS in 25, Devic's disease in $4,^{[5,6,10]}$ recurrent transverse myelitis in $1,{ }^{[11]}$ acute myelitis in $3,{ }^{[8]}$ and acute disseminated encephalomyelitis in $2 .{ }^{[8]}$ Co-occurrence of other autoimmune disorders such as systemic lupus erythematosis, ${ }^{[12]}$ Graves's disease ${ }^{[13]}$ iritis, asthma,

\begin{tabular}{|c|c|c|c|c|c|}
\hline Age & Gender & $\begin{array}{l}\text { Age at } \\
\text { onset } \\
\text { of MS }\end{array}$ & $\begin{array}{c}\text { Age at } \\
\text { diagnosis } \\
\text { of MS }\end{array}$ & $\begin{array}{l}\text { Age at } \\
\text { onset } \\
\text { of } M G\end{array}$ & $\begin{array}{c}\text { Age at } \\
\text { diagnosis of } \\
\text { MG }\end{array}$ \\
\hline 25 & $\mathrm{~F}$ & 23 & 23.5 & 16 & 24 \\
\hline 41 & $\mathrm{~F}$ & 31 & 37 & 40 & 40 \\
\hline 55 & $\mathrm{~F}$ & 51 & 51 & 52 & 52 \\
\hline 40 & $\mathrm{~F}$ & 38.5 & 39 & 38 & 38 \\
\hline 26 & $\mathrm{~F}$ & 19 & 21.5 & 21 & 21 \\
\hline
\end{tabular}

MG - Myasthenia gravis; MS - Multiple sclerosis poly-arthritis, ${ }^{[9]}$ and antiphospholipid antibodies ${ }^{[14]}$ have been reported in patients with both MS and MG. None of our patients had any other autoimmune diseases.

All the six patients including the one excluded for calculating prevalence rate had fulfilled the McDolad's diagnostic criteria for definite MS. All the six patients had classical magnetic resonance findings; however, none had lumbar puncture as it was not a mandatory criterion for the diagnosis of MS. However, the diagnosis of MG was based on the fluctuating clinical features, positive RNS, and edrophonium test. It is a well recognized fact that fluctuations in symptoms is common in patients with MS and there may be a non-specific response to anti-cholinesterase medication especially in those with fatigue. However, in our patients the distribution of fluctuating weakness was ocular or bulbar which is not a common feature in MS. The other important issue was that none of the six patients was positive for acetylcholine receptor antibodies. In this series most of the patients had fluctuating ocular symptoms and the antibodies may not be always detectable in ocular form of MG. Facilities for testing muscle specific tyrosine kinase (MuSK) antibody, and single fiber EMG were not available at our center. It is quite possible that we might have diagnosed more cases of MG using these two tests and the prevalence of MG among patients with definite MS would have been much higher. Even then with these limitations the estimated frequency (291/100000) of MG among patients with definite MS in this study was much higher than the frequency of MG in general population (5-15/100000).

In the series from British Colombia, ${ }^{[10]}$ MS preceded MG in five of the eight patients by 4 to 9 years and in three patients MG preceded MS by 6 to 8 years. In our study in three patients MS preceded the onset of MG symptoms. The first manifestation of MG in all of the patients in the British Colombia study ${ }^{[10]}$ was ocular (ptosis in four, dipplopia in two, and both of them together in remaining two). In our study, three patients had ocular manifestations as the initial symptom, one had both ocular and bulbar symptoms and the remaining patient had fluctuating dysphonia. The first presenting features of MS in the British Colombia study were: Optic neuritis

\begin{tabular}{|c|c|c|c|c|c|c|c|c|}
\hline Patient ID & $\begin{array}{l}\text { Initial MS } \\
\text { symptom }\end{array}$ & MS course & $\begin{array}{l}\text { EDSS } \\
\text { score }\end{array}$ & $\begin{array}{l}\text { Initial MG } \\
\text { symptom }\end{array}$ & RNS & $\begin{array}{l}\text { Ach receptor } \\
\text { antibodies }\end{array}$ & $\begin{array}{l}\text { Tensilone } \\
\text { test }\end{array}$ & $\begin{array}{l}\text { Thymectomy thymus } \\
\text { histology }\end{array}$ \\
\hline 1 & $\begin{array}{l}\text { Right lower limb } \\
\text { paresthesia }\end{array}$ & $\mathrm{RR}$ & 2.5 & Dysphonia & Decremetal & Negative & Positive & No \\
\hline 2 & Optic neuritis & RR & 1 & Ptosis, & Decremetal & Negative & Positive & No \\
\hline 3 & Optic neuritis & $\mathrm{RR}$ & 2 & Ptosis, dipplopia & Decremetal & Negative & Positive & $\begin{array}{l}\text { Yes, Thymic follicular } \\
\text { hyperplasia }\end{array}$ \\
\hline 4 & $\begin{array}{l}\text { Left lower limb } \\
\text { paresthesia }\end{array}$ & $\mathrm{RR}$ & 1.5 & Ptosis, dipplopia & Decremetal & Negative & Positive & Yes, Thymic hyperplasia \\
\hline 5 & Facial palsy & $\mathrm{RR}$ & 1.5 & Ptosis dysartheria & Decremetal & Negative & Positive & No \\
\hline
\end{tabular}

MG - Myasthenia gravis; MS - Multiple sclerosis; RNS - Repetitive nerve stimulation; EDSS - Expanded disability status scale 
in 5 , facial palsy in 1 , paresthesia in 1 , and weakness, bladder and balance disturbances in 1 . In our study the first presenting features of MS were: Optic neuritis in 2, limb paresthesia in 2 and facial palsy in 1 . The course of MS was relapsing-remitting (RR) in both our study and the British Colombia study. EDSS score in the British Colombia study were high and ranged between 0.5 and 6 and in our study the scores ranged between 1 and 2.5; in our study the disease was milder.

Three of the patients in the British Colombia study had thymectomy and biopsy showed thymic hyperplasia. In two of our patients who had thymectomy, biopsy showed thymic hyperplasia. It is possible that thymectomy may alter the immune system and may predispose to the development of MS. ${ }^{[8,15,16]}$ This argument may not be relevant in both our patients as MS was the initial event. Onset of MG in MS patients receiving beta-interferon ${ }^{[7,17]}$ or glatiramer acetate ${ }^{[18]}$ have been reported, but none of our patients were on such disease modifying treatments. If the association between MS and MG is true it is quite possible that thymectomy may be of value for MS. ${ }^{[19]}$

MS patients with undue fatigue or fluctuating features should be investigated for MG. Awareness of this possible association would lead to early diagnosis of ocular MG as the ocular manifestation are more common in patients with MS and MG. Early diagnosis and institution of appropriate treatment may prevent the progression of the disease to generalized MG and may also lower the morbidity.

\section{Acknowledgments}

We appreciate contribution of Dr.Christopher H. Hawkes (Essex Neuroscience Centre, Queens Hospital, Rumford, UK) for his revision and precious comments.

\section{References}

1. Donmez B, Ozakbas S, Oktem MA, Gedizlioglu M, Coker I, Genc A, et al. HLA genotypes in Turkish patients with myasthenia gravis: Comparison with multiple sclerosis patients on the basis of clinical subtypes and demographic features. Hum Immunol 2004;65:752-7.
2. Saadatnia M, Etemadifar M, Maghzi AH. Multiple sclerosis in Isfahan, Iran. Int Rev Neurobiol 2007;79:357-75.

3. Oosterhuis HJ. The natural course of myasthenia gravis: A long-term follow up study. J Neurol Neurosurg Psychiatry 1989;52:1121-7.

4. Robertson NP, Deans J, Compston DA. Myasthenia gravis: A population based epidemiological study in Cambridgeshire, England. J Neurol Neurosurg Psych 1998;65:492-6.

5. Furukawa Y, Yoshikawa H, Yachie A, Yamada M. Neuromyelitis optica associated with myasthenia gravis: Characteristic phenotype in Japanese population. Eur J Neurol 2006;13:655-8.

6. Kister I, Gulati S, Boz C, Bergamaschi R, Piccolo G, Oger J, et al. Neuromyelitis optica in patients with myasthenia gravis who underwent thymectomy. Arch Neurol 2006;63:851-6.

7. Dionisiotis J, Zoukos Y, Thomaides T. Development of myasthenia gravis in two patients with multiple sclerosis following interferon beta treatment. J Neurol Neurosurg Psychiatry 2004;75:1079.

8. Gotkine M, Fellig Y, Abramsky O. Occurrence of CNS demyelinating disease in patients with myasthenia gravis. Neurology 2006:12;67:881-3.

9. Somer H, Muller K, Kinnunen E. Myasthenia gravis associated with multiple sclerosis. Epidemiological survey and immunological findings. J Neurol Sci 1989;89:37-8.

10. Isbister CM, Mackenzie PJ, Anderson D, Wade NK, Oger J. Co-occurrence of multiple sclerosis and myasthenia gravis in British Columbia. Mult Scler 2003;9:550-3.

11. Lindsey JW, Albers GW, Steinman L. Recurrent transverse myelitis, myasthenia gravis, and autoantibodies. Ann Neurol 1992;32:407-9.

12. Tola MR, Casetta I, Granieri E, Caniatti LM, Monetti VC, Pascarella R. Systemic lupus erythematosus related recurrent transverse myelitis in a patient with myasthenia gravis and multiple sclerosis. Eur Neurol 1996;36:327-8.

13. Bixenman WW, Buchsbaum HW. Multiple sclerosis, euthyroid restrictive Grave's ophthalmopathy, and myasthenia gravis. A case report. Graefes Arch Clin Exp Ophthalmol 1988;226:168-71.

14. Fujioka T, Ishida T, Kurihara T, Kinoshita M. A case of myasthenia gravis associated with multiple sclerosis and positive anticardiolipin antibodies. Rinsho Shinkeigaku 1993;33:572-4.

15. Alemany-Rodriguez MJ, Aladro Y, Amela-Peris R, Pérez-Viéitez MC, Reyes-Yáñez MP, Déniz-Naranjo MC, et al. Autoimmune diseases and multiple sclerosis. Rev Neurol 2005;40:594-7.

16. Nakamura Y, Takahashi M, Yorifuji S, Hazama T, Taru S. A case of multiple sclerosis occurred after thymectomy in myasthenia gravis. Rinsho Shinkeigaku 1986;26:937-40.

17. Blake G, Murphy S. Onset of myasthenia gravis in a patient with multiple sclerosis during interferon-1b treatment. Neurology 1997;49:1747-8.

18. Frese A, Bethke F, Ludemann P, Stögbauer F. Development of myasthenia gravis in a patient with multiple sclerosis during treatment with glatiramer acetate. J Neurol 2000;247:713.

19. Flechter S, Vardi J, Just I, Arnon R, Teitelbaum D. Thymectomy and chronic relapsing experimental allergic encephalomyelitis in guinea pigs. Arch Neurol 1984;41:1158-60.

Accepted on 6-10-2009

Source of Support: Nil, Conflict of Interest: None declared. 